\title{
RETHINKING THE PHILIPS PAVILION THROUGH ROBOTIC HOT WIRE CUTTING.
}

\section{An experimental prototype}

\author{
Pedro Filipe Martins ${ }^{1}$, Sandra Nunes ${ }^{2}$, Paulo Fonseca de Campos ${ }^{3}$, \\ José Pedro Sousa ${ }^{4}$ \\ ${ }^{1,4}$ Faculty of Architecture, University of Porto + DFL/CEAU/FAUP ${ }^{2}$ Faculty of \\ Engineering, University of Porto ${ }^{3}$ Faculty of Architecture and Urbanism, Univer- \\ sity of São Paulo \\ ${ }^{1,4}\left\{\right.$ pcarvalho|jsousa\}@arq.up.pt ${ }^{2}$ snunes@fe.up.pt ${ }^{3}$ pfonseca@usp.br
}

\begin{abstract}
The Philips Pavilion by Le Corbusier and Jannis Xenaquis was a landmark project in thin shell concrete construction, only made possible by an experimental precasting strategy that deeply defined the architectural character of the hyperbolic paraboloid surfaces of the pavilion. Using this historic precedent this research presents a reinterpretation of the design of the Philips Pavilion, specifically tailored for Robotic Hot Wire Cutting technologies and a layered mold system, combining speed and material optimization towards more sustainable concrete construction processes. By documenting the realization of an experimental prototype at a 1:2 scale, this paper demonstrates the feasibility of the proposed strategy and its value in comparison with existing construction scale digital fabrication technologies for concrete.
\end{abstract}

Keywords: Digital Fabrication, Concrete, Robotic Hot Wire Cutting, Philips Pavilion

\section{INTRODUCTION \\ Background}

In the second half of the XXth century, several remarkable concrete thin shell structures were built, featuring curved geometries of increasing complexity. Such structures were built mostly through analogic and empiric design and construction processes, which not only were complex and costly, but also imposed significant design constraints and sustainability issues, particularly in the production of the necessary scaffolding and formwork for their realization.
One such example was the Philips Pavilion, designed by Le Corbusier and Yannis Xenaquis for the 1958 World Exhibition in Brussels, featuring a set of extremely curved hyperbolic paraboloid surfaces. The pavilion's construction was, at the time, only made possible through an unconventional construction process. The selected strategy used hand-made sand molds for the prefabrication of approximately 2000 rhomboid concrete panels composing the 9 ruled concrete surfaces of the pavilion (Duyster 1959) (Figure 1). 


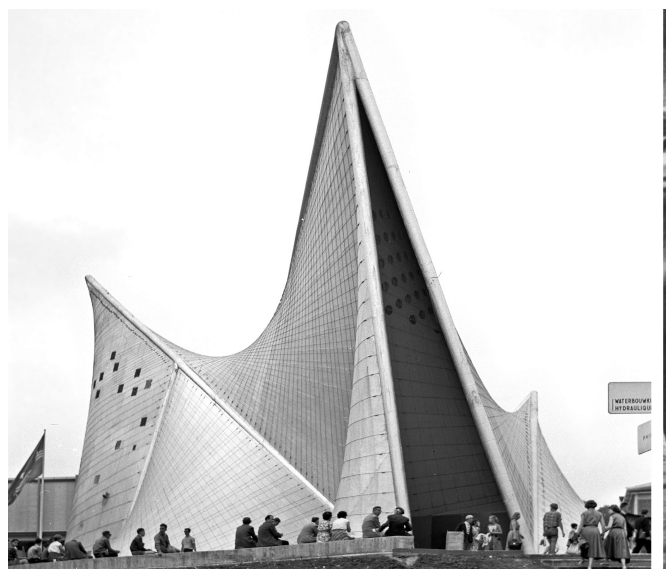

Although ultimately successful, it was an expensive and labor intensive process, imparting specific design constraints on the architecture of the Philips Pavilion. Notwithstanding the great commercial success of the pavilion, it was demolished soon after the exhibition ended.

Subsequently, there has been some research pointing to strategies for rebuilding the Philips Pavilion using modern construction technologies, which generally dismiss the original prefabricated subdivision strategy that gave it its character, for more efficient construction strategies. Methods such as in-situ concreting, large prefabricated shells and sprayed concrete using membrane formworks can be found in existing literature (Pronk 2007; Nijse 2008). Although as of yet there hasn't been an actual reconstruction of the pavilion, these demonstrate not only the continuing architectural relevance of the Philips Pavilion but also the challenge that erecting such a structure still presents today.

During the last decade, the increased demand for complex concrete structures stemming from the development of digital design and fabrication technologies has been accompanied by an increased awareness for the issue of sustainability in construction. Though several new methods for the production of freeform concrete surfaces have been devel-

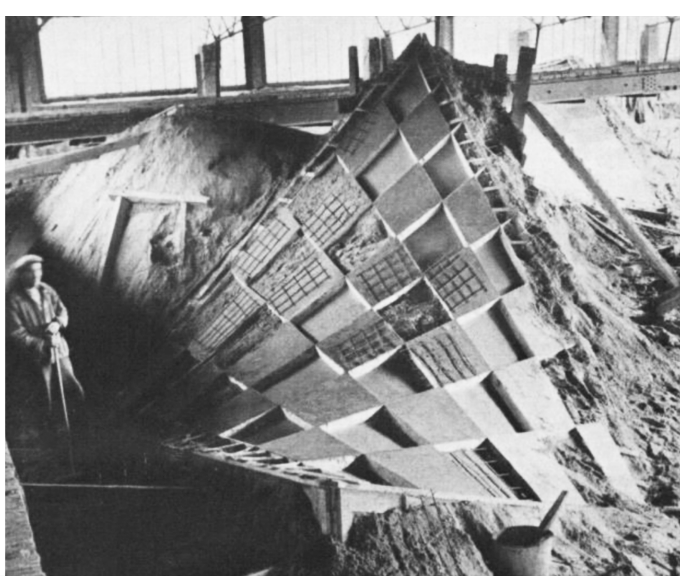

oped that could be effectively applied to a potential reconstruction of the pavilion, the production of such large-scale and complex constructions still present economic and sustainability problems with design consequences that should be addressed within the field of architectural research.

\section{Objectives}

This paper proposes to address these issues by rethinking the construction of the Philips Pavilion through the use of tailored digital fabrication technologies for the production of complex ruled surfaces. Considering the ruled geometry of the pavilion and the original precast strategy, we believe that Robotic Hot Wire Cutting (RHWC) can not only improve the efficiency and sustainability of the construction process, but can also introduce new possibilities in the design of the pavilion, maintaining the tectonic link between construction and design that was already present in the original work.

Within this framework, the work presented in this paper focused on 2 main goals:

- develop a layered formwork strategy tailored for RHWC, based on the sequential cutting of large Expanded Polystyrene (EPS) blocks, optimizing material usage and pro-
Figure 1

Philips Pavilion (1958); sand mound formwork for ruled surface panels (1957) 
duction processes, while maintaining its economical and industrial viability.

- define and explore a new design space for the pavilions' panel geometry, emerging from the properties of this digital fabrication process and the geometrical needs of the mold strategy.

\section{STATE OF THE ART}

\section{Current industrial formwork solutions for freeform concrete}

Contemporary industrial solutions for the construction of complex curved geometries in concrete generally depend on CNC cutting and milling technologies for formwork production of either in-situ or precast structures. Such technologies can be employed in manually assembled wood formwork, using precut elements such as guides, templates or entire formwork sections. Examples of works using this strategy can be found in the Rolex Learning Center (SANAA, 2009) and the Waal Bridge Extension (Zwart \& Jansma, 2015).

Large-scale, multiple-axis CNC milling can also be used in the production of EPS (expanded polystyrene) foam molds either as inserts in traditional in-situ formwork systems, as was the case of the Spencer Dock Bridge (Amanda Levete, 2009), or as stand-alone molds for precast components, as seen in the Zollhof Towers (Gehry, 1999) and, more recently in the Louisiana State Museum (Trahan Architects, 2013).

Although CNC milling still presents important advantages for the production of complex and customized formwork (i.e. its geometric freedom and versatility), it also presents several drawbacks. The need for smooth casting surfaces and the inherently slow process of incremental subtraction of material that defines CNC milling result in its two major disadvantages: 1. overall high machining times and costs and 2. large volumes of consumed and wasted materials. Considering these drawbacks, CNC milling is usually reserved for works of exceptional character and corresponding budgets, such as the ones previously mentioned.

Alternatively, the use of Robotic Hot Wire Cutting (RHWC) of EPS foam blocks for the production of concrete molds has been explored in several academic prototypes and has also been tested in largescale commercial implementations, such as the insitu formwork for Kirk Kapital Headquarters (Olafur Eliasson, 2018). For the exclusive production of ruled surfaces in concrete, RHWC has now been generally demonstrated as a viable alternative to CNC milling with reduced costs, operation times and waste volumes (Søndergaard 2017). More recent developments are now focusing on technological improvements, such as multiple robot setups and more flexible end-effectors such as hot-blades and abrasive wires (Søndergaard 2016).

As its still far from a broadly accepted process in industry, the authors believe that there is still room for increased sustainability, optimization and exploration of RHWC in relevant case studies. Particularly, this research aims to shift focus from the development of technological tools, to the exploration of specific molding processes related to RHWC and their potential impact on the constructive and architectural aspects of the Philips Pavilion.

\section{RETHINKING THE PHILIPS PAVILION}

In order to assess the possibility of rebuilding the complex concrete surfaces of the Philips Pavilion through the use of a tailored robotic fabrication process and mold strategy, an experimental prototype was devised which will be documented and discussed in this section. After consideration of the 9 hyperbolic paraboloid surfaces composing the pavilion, one was selected as a case study for this research (figure 3.2). From it, new subdivision designs were explored and production, using a layered mold strategy, was compared to a CNC milling scenario. Finally, a prototype consisting of a 1:2 scale section, composed of $950 \times 50 \times 5 \mathrm{~cm}$ precast micro-concrete panels was built, through the use of Robotic Hot Wire Cutting. 

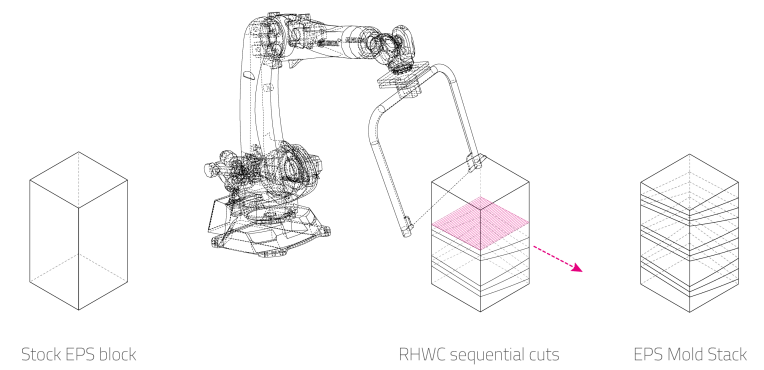

Figure 2

Layered mold design logic for RHWC

\section{Layered Mold strategy for RHWC}

The logic of layered or stacked molds for concrete production is not an entirely new process. In academia, there have been examples of the use of similar logics, applied to concrete, using different fabrication processes (Enrique 2016) or directly to other materials such as ceramics (Mankouche 2012). In the precast industry there is also the specific precedent of mold batteries for the large-scale production of identic flat, double-sided concrete panels. The central aspect of this logic in all cases is that elements can be cast (or cut) side by side, with one side of one element being coincident with another side of the next. This logic can be particularly relevant when used in conjunction with RHWC.

One of the main advantages of RHWC over traditional subtractive technologies (i.e. milling) for mold fabrication in foam like materials (EPS) is the preservation of excess material when cutting a surface. Although this feature is a staple of RHWC, the general usage of such molds has been similar to other subtractive processes - one section per mold. An alternative to this logic is the creation of successive cuts, subdividing a stock EPS block into several layers. Applying this concept to mold design allows for the creation of a layered mold of stacked pieces with varying geometry, from one single block of stock material.

Depending on the shape geometry and the positioning of a panel surface within the stock material, this process can be optimized for a minimum of 1 cutting operation per panel surface, foregoing the need for perimeter cuts, if all edges of the panel geometry are coincident with the stock material sides. If this condition is met, the mold layers can then be separated with a specific distance, leaving voids to be cast with concrete (figure 2 ).

\section{Surface design for RHWC and a layered mold strategy}

The original panel design for the precast panels of the Philips Pavilion was constrained by the necessity of having panel boundaries following the ruling directions of the overall ruled surface. This was imperative for workers to have straight wood guides for the manual description of the ruled surfaces of each panel. Using these guides and an underlying sand mound (also molded following surface rulings), the workers could cast concrete in the subdivided voids and accurately describe the necessary panel geometry with simple wood beams (Duyster 1959).

In the layered mold strategy developed in this work the relevant geometrical constrain is the coinciding projection of the panel surfaces within a boundary volume. With this fabrication logic in mind, a geometrical process was developed for designing viable paneling subdivisions that could be subsequently grouped in a vertical stack with coinciding edge projections. This was achieved simply through the projection of a regular tessellation (a) onto the ruled surface (b), in a specific direction (d) (figure 3.1). Using this process, the shape of the tessellation dictates the stack section geometry (ex. square), while 
Figure 3

Surface subdivision logic; subdivision design studies; RHWC and CNC Milling comparison
1. SUBDIVISION LOGIC

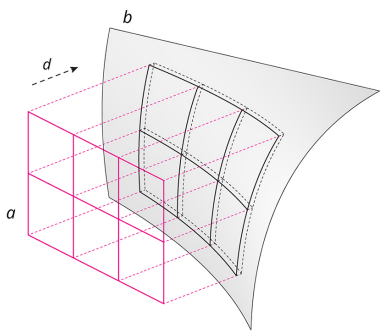

Projected regular subdivision in chose direction

2. SUBDIVISION DESIGN

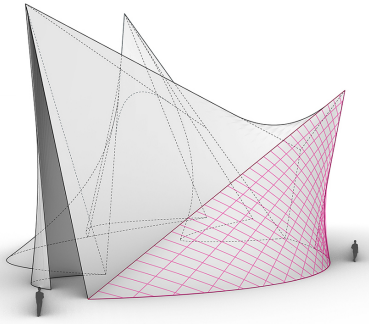

Selected hyperbolic paraboloid surface and subdivision design

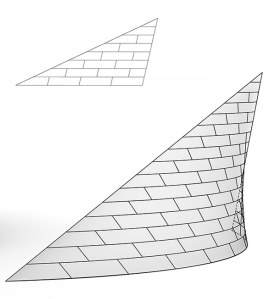

Subdivision test 2

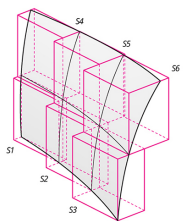

Distorted panel geometry in equal bounding boxes

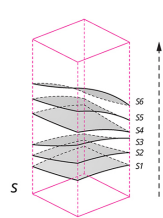

Collected panel surfaces
3. PRODUCTION SIMULATION

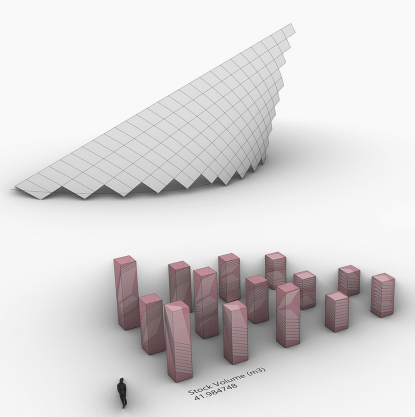

RHWC + Layered EPS molds

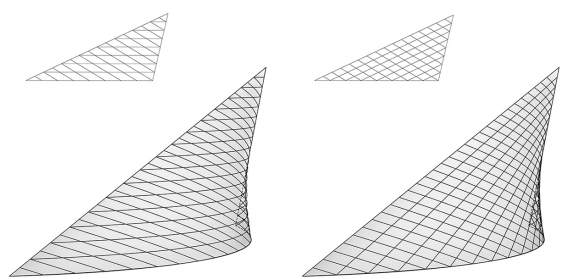

Subdivision test 3

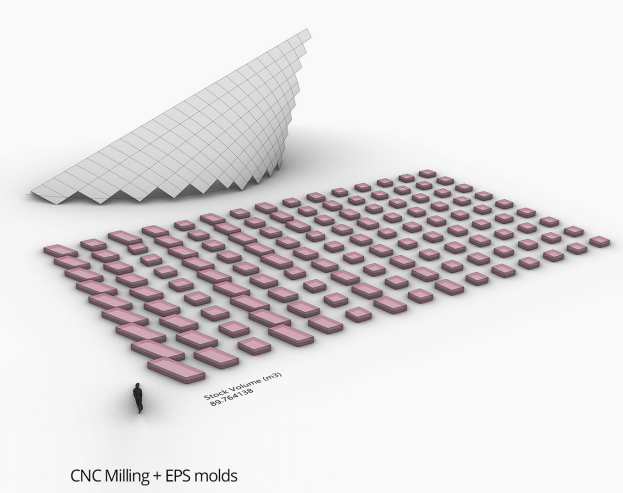

Matter - DIGITAL PRODUCTION AND ROBOTICS 2 - Volume 3 - eCAADe 37 / SIGraDi 23 | 239 
the projection direction dictates the panel's shape on the surface and its fixed position inside the stack.

This strategy established from the onset a different possible vocabulary for the pavilion surfaces, in line with the existing architectural logic of prefabrication but with an expanded design space featuring a new set of guiding principles and rules, directly linked to this digital fabrication strategy and not limited by the ruling directions.

Using this method, the surface subdivision was explored in several solutions, tailored for different purposes. Goals such as panel size and curvature (large vs small), projected shape geometry (square, rectangular, losangular, triangular) and boundary variation minimization (recursive subdivision) were explored (figure 3.2). On a design note, considering the original strict subdivision that exacerbated the rulings of the parabolic hyperboloid geometry, in this reinterpretation the authors favored solutions with evidently curved boundaries.

Within these parameters, a solution was chosen that combined the ease of production of regular square section EPS material blocks with ruled panel boundaries in one direction and curved boundaries in another direction, emphasizing the curvature of the ruled surface and distinguishing it from the original ruled subdivision (figure 3.2).

To simulate and evaluate the production of the selected solution, a simple algorithm was developed that automated the grouping (along ruling directions) and translation of the 135 individual panels into stacks of 10 panels each, with a minimum separation of $10 \mathrm{~cm}$ between panel surfaces to ensure a suitable EPS thickness for the molds. No further optimization of distribution and nesting was attempted.

The entire process was evaluated and compared to a CNC milling scenario of individualized molds. For this purpose, it was equated that the CNC milled molds would be tailored for minimization of milled height and the panels were situated in the stock material with this consideration, including a minimum of $20 \mathrm{~cm}$ of EPS height for stabilization and an offset of $5 \mathrm{~cm}$ for boundary walls. This resulted in 14 stacks for the case of the layered molds and 135 individual elements for the case of CNC milled molds. Analyzing the results for material volume, the first case revealed an expenditure of approximately $42 \mathrm{~m} 2$ of EPS, in comparison with approximately $89 \mathrm{~m} 2$ for the same surface area in the CNC milling scenario (figure 3.3).

\section{Robotic fabrication and concrete produc- tion process}

After scaling down the selected prototyping area of $3 \times 3$ panels to a 1:2 scale, the same grouping and surface translation process developed earlier was employed for a stock material geometry of $50 \times 50 \times 100 \mathrm{~cm}$ EPS blocks (figure 4).
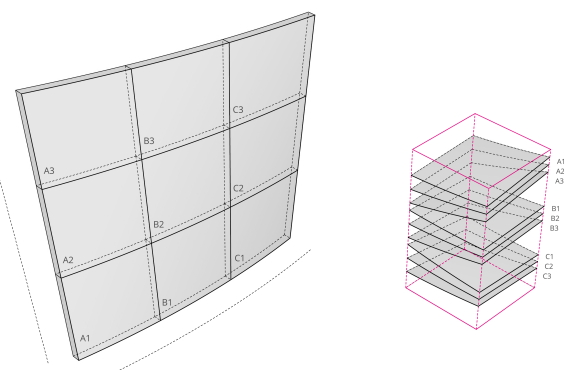

For the robotic production of the molds, simulation and toolpath generation was done in Grasshopper for Rhinoceros, using the Kuka|prc plugin. The prototype stack was cut using a 6-axis Kuka KR 120 industrial robot, equipped with a large-scale hot wire end-effector and a maximum cutting width of $85 \mathrm{~cm}$. The cutting sequence was completed in a straightforward process of 10 cuts, one per surface, without any issues arising from this process. The entire sequence was concluded in approximately 15 minutes, corresponding to the relatively slow speed (aprox. $1 \mathrm{~cm} / \mathrm{s}$ ) and temperatures used to ensure correct cutting geometry.

For the construction of the molds, the layered stack was placed sideways, separated with the desired panel width $(5 \mathrm{~cm})$ and assembled inside a form-
Figure 4

Prototype panel model; Cutting surfaces in stack 
Figure 5

RHWC EPS stack

after cutting

process; Assembled

test prototype from

mold parts.
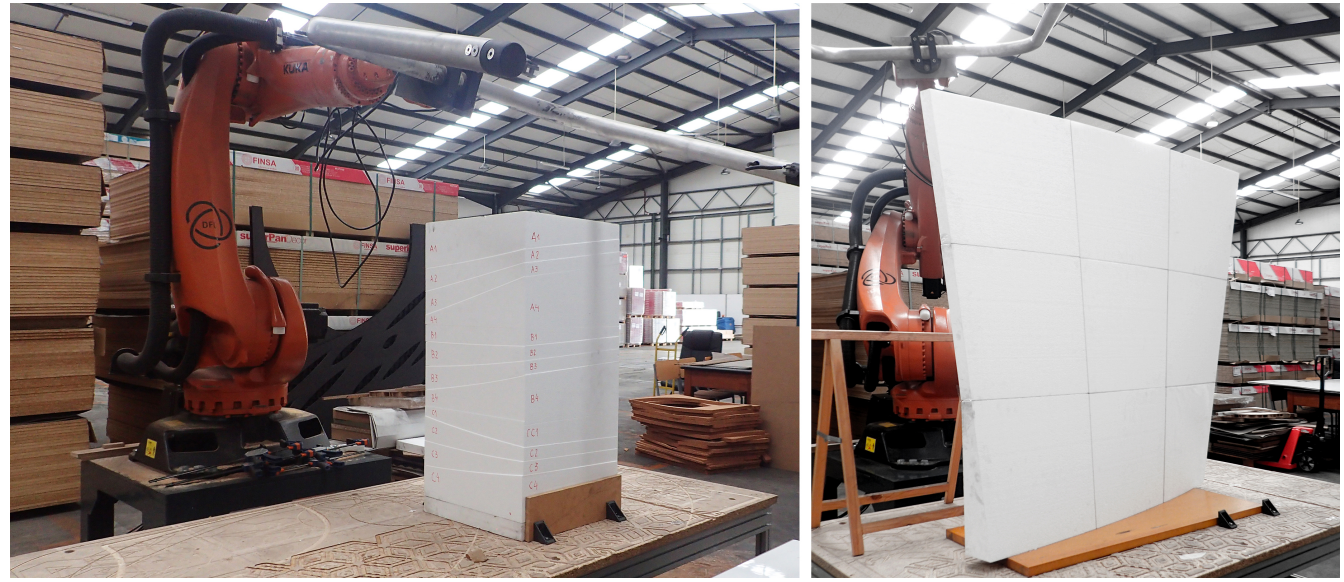

work plywood shell, leaving the voids to be poured with concrete. The plywood shell was closed and clamped, leaving the top side open for vertical casting in the voids left by the EPS elements. After initial experiments, this mold design was improved with 2 fixed subdivisions, dividing the panels in groups of 3 to increase EPS resistance to concrete casting pressures and prevent any buckling from occurring inside the molds. (figure 6)

In preparation for the casting process, no postprocessing was done on the EPS surfaces and a simple coating of demolding oil was used to ensure a correct casting and demolding of the panels and to minimize the overall production time and costs.

Considering the closed mold design with the desired panel geometry and the difficulties of vibrating and applying regular reinforcement meshes, a self-compacting concrete mix was used, consisting of grey Portland cement, fine aggregates and fillers, combined with polyprolylene fibers for reinforcement.

Concrete pouring was executed sequentially, in groups of 3, in order to further prevent differences in pressure inside the individual voids and minimize the lateral pressure of the concrete on the EPS molds parts. The panels were demolded after 8 days of cur- ing time and assembled in their final disposition, confirming the desired geometry of the Philips Pavilion prototype.

\section{DISCUSSION AND CONCLUSIONS}

In the development of the described research, different constraints and characteristics of the proposed process became apparent that are discussed here, before conclusions are drawn.

Regarding the geometric constraints of the layered strategy and required design, some consideration should be taken in selecting the projection direction of the subdivision in order to avoid excessively vertical panels inside the stock material and optimize material usage. Also, the process assumes (as the original construction method), that the panel sides are not perpendicular to the surface curvature. This evidently prevents its usage in stereotomic assemblies, where such characteristic is fundamental, without using variable section stacks. These inherent constrains clearly position this strategy between the complete dependency on ruled boundaries of the original casting solution and the complete geometrical freedom of the CNC milling process. Moreover, although the non-square EPS blocks necessary for some of the studied surface subdivision cases would 

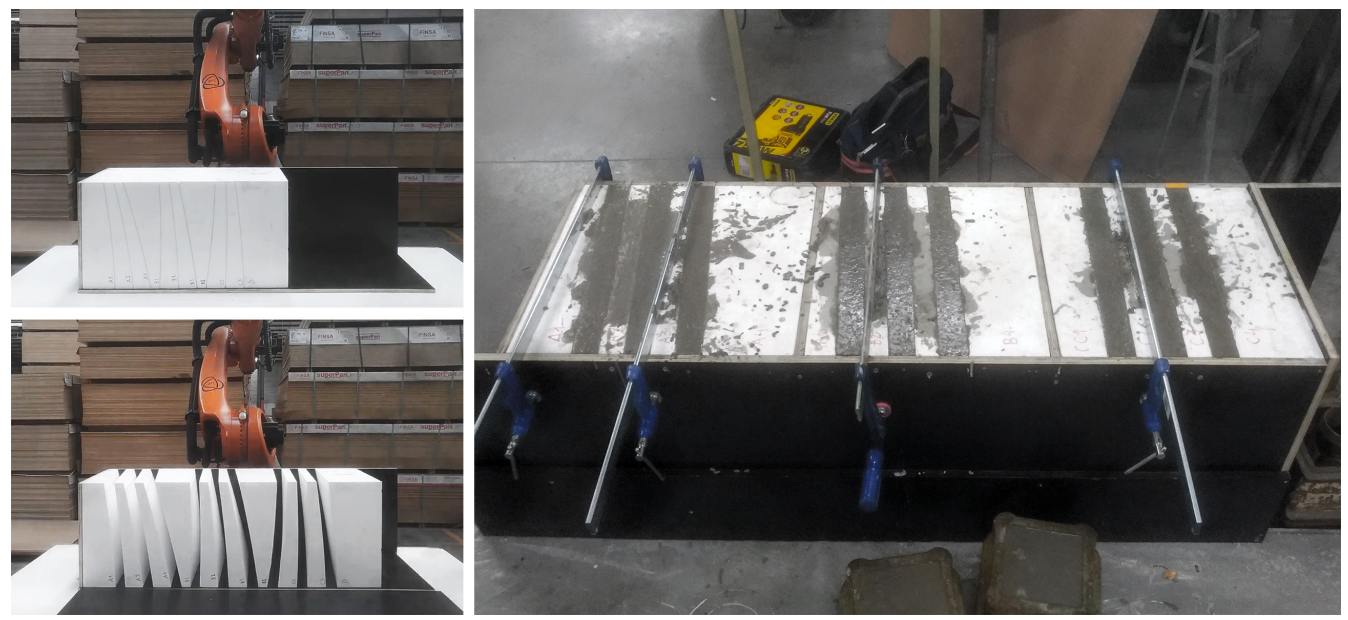

Figure 6

EPS mold

preparation; closed

mold after casting

complicate formwork design, the advantage of building the entirety of the ruled surface, using only 2 types of mold stacks could represent considerable benefits in terms of construction optimization.

Regarding the Robotic Hotwire Cutting, as discussed before, the process presented considerably reduced times when compared to normal CNC machining for the same surface area. Considering approximately $120 \mathrm{~min} / \mathrm{m} 2$ to $240 \mathrm{~min} / \mathrm{m} 2$ for CNC milling (depending on desired surface smoothness) and the extrapolation of $240 \mathrm{~s} / \mathrm{m} 2$ for RHWC, this represents a 30 to 60 fold decrease in fabrication time for RHWC. These times were somewhat offset by the added time and manual labor of assembling of the mold stacks into the plywood formworks, leading to consider the necessity of improving this stage of the process. Nevertheless, the increased output of the process in an industrial setting would mitigate this aspect and should not be comparable with full manual assemblies of wood and sand formwork of the original process.

As expected from previous experiments, the surface quality of the concrete panels (figure 7) was not comparable with precast panels using other strategies, such as regular wood formwork or molding ta- bles using membranes, due to the EPS porosity. Nevertheless, improvement measures can be taken to achieve the same qualities, through the use of EPS coatings, without compromising the overall process. Moreover, the use of the closed molds with lateral panel sides facing the plywood panels, resulted in smooth and precise boundaries in the finished panels.

In conclusion, we believe that the RHWC process in conjunction with the layered mold system presents clear advantages to traditional mold systems and several other digital fabrication strategies. It leverages the speed and efficiency of Robotic Hot Wire Cutting with the ability to rationalize curved panel subdivisions to maximize production efficiency, thus attaining an increased sustainability by decreasing material volume waste by roughly $50 \%$ when compared with current milling strategies. At the technical level, other advantages can also be found, namely 1) the reduction of processing times (and output volumes per square meter), 2) transportation and storage costs (through volume optimization), 3) ease of integration with existing precast practices, without the need for other complex digital fabrication procedures. All such 
Figure 7

Assembly of 4

finished panels

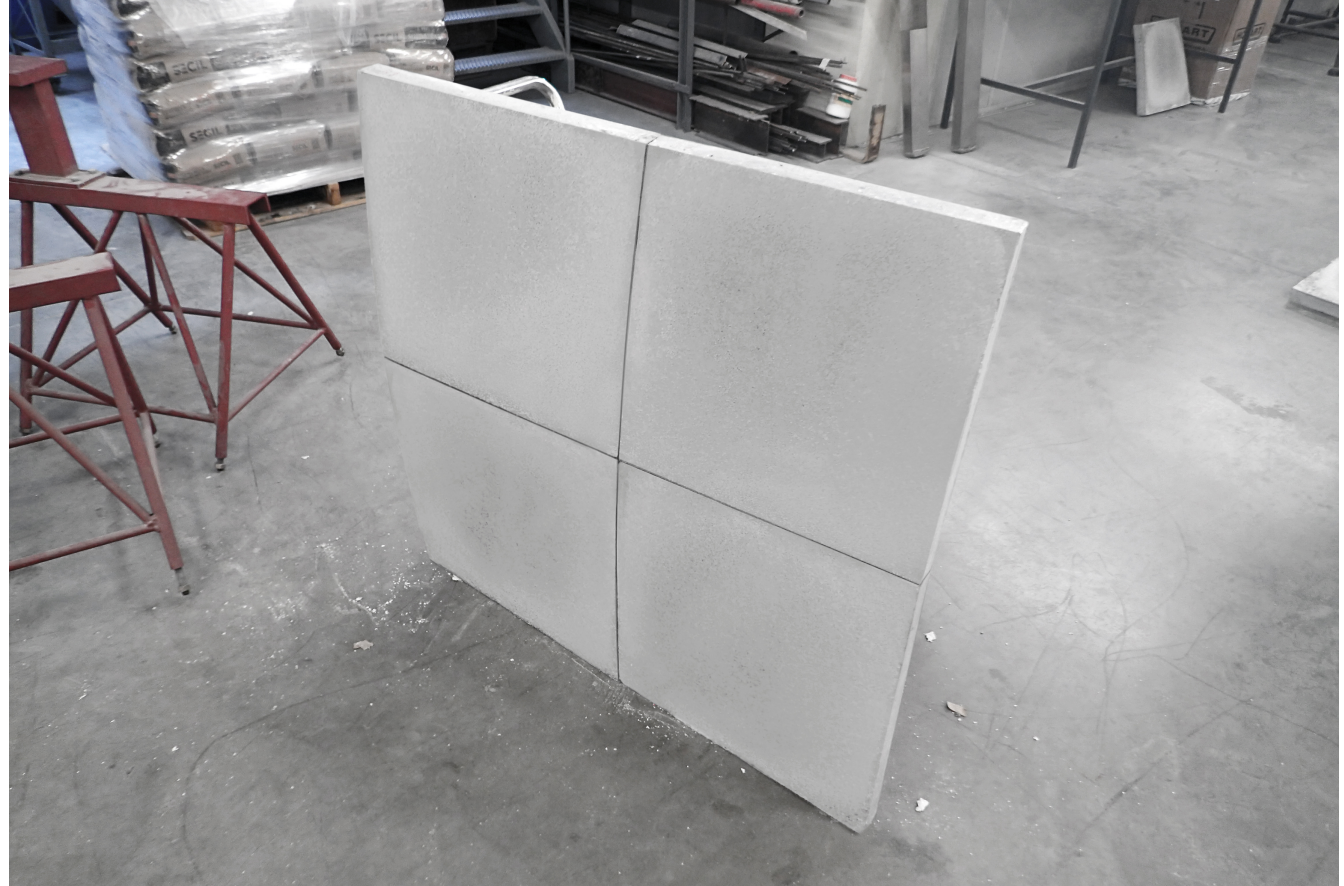

advantages represent important chances for an increased democratization of digital fabrication strategies in current construction practice.

Finally, past experiments in rebuilding the Philips Pavilion were mostly focused on the construction of continuous or (mostly continuous) hypar surfaces in concrete. While this was certainly a possibility considered by the original architects, we believe that the final built pavilion expressed a more nuanced story of technological innovation in precast concrete construction, which fueled the particular architectural expression and tectonic relevance of the Philips Pavilion.

At this architectural level, this research demonstrates a possibility of rebuilding the Philips Pavilion within this spirit, maintaining the original tectonic character of its ruled, prefabricated surfaces in a reinterpreted design, specifically for RHWC. On a broader scale, this research shows how digital fabrication technologies can be configured for enhanced sustainability and efficiency, while also driving architectural language in coherent tectonic expressions, maintaining strong relations between construction and design.

\section{ACKNOWLEDGMENTS}

The authors would like to thank all the Digital Fabrication Lab (DFL) team and the FEUP/CONSTRUCT team and support staff for the help in developing the work present in this paper.

\section{REFERENCES}

Søndergaard, A, A 2016 'Robotic hot-blade cutting', RobArch 2016: Robotic Fabrication in Architecture, Art and Design, Sydney, p. 150-164 
Duyster, HC 1959, 'Construction of the Pavilion in Prestressed Concrete', Philips Technical Review, 20, pp. 27-36

Enrique, L and Schwartz, J 2016 'CASTonCAST Shell Structures: Realisation of a 1: 10 Prototype of a post-tensioned shell structure from precast stackable components', Advances in Architectural Geometry 2016, Zurich, pp. 364-377

Mankouche, S and Bard, J 2012 'Morphfaux: Probing the Proto-Synthetic Nature of Plaster Through Robotic Tooling, Proceedings of the 32nd Annual Conference of the Association for Computer Aided Design in Architecture (ACADIA), San Francisco, pp. 177-186

Nijse, R 2008, 'Rebuilding Le Corbusier's World Exhibition Pavilion; The Poème Electronique in Brussels, 1958', in Walraven, J and Stoelhorst, D (eds) 2008, TailorMade Concrete Structures, Taylor and Francis

Pronk, A, Houtman, R and Afink, M 2007 'The Reconstruction of the Philips Pavilion', Proceedings of the conference Sources of Architectural Form, Kuwait

Søndergaard, A and Feringa, J 2017 'Scaling architectural robotics: construction of the Kirk Kapital headquarters', Proceedings of Fabricate 2017, pp. 264-271 